\title{
Propulsion Trade Studies for Spacecraft Swarm Mission Design
}

\author{
Andres Dono \\ Millennium Engineering \\ and Integration Services \\ NASA Ames Research Center \\ Moffett Field 94035 \\ 650-604-2025 \\ andres.donoperez@nasa.gov
}

\author{
Laura Plice, Joel Mueting \\ Metis Technology Solutions \\ NASA Ames Research Center \\ Moffett Field 94035 \\ 650-604-2438 \\ laura.plice@nasa.gov \\ joel.r.mueting@nasa.gov \\ Michael Ho \\ Stinger Ghaffarian Technologies \\ NASA Ames Research Center \\ Moffett Field 94035 \\ 650-604-6375 \\ michael.ho@nasa.gov
}

\author{
Tracie Conn \\ NASA Ames Research Center \\ Moffett Field 94035 \\ 650-604-5365 \\ tracie.conn@nasa.gov
}

\begin{abstract}
Spacecraft swarms constitute a challenge from an orbital mechanics standpoint. Traditional mission design involves the application of methodical processes where predefined maneuvers for an individual spacecraft are planned in advance. This approach does not scale to spacecraft swarms consisting of many satellites orbiting in close proximity; non-deterministic maneuvers cannot be preplanned due to the large number of units and the uncertainties associated with their differential deployment and orbital motion. For autonomous small sat swarms in LEO, we investigate two approaches for controlling the relative motion of a swarm. The first method involves modified miniature phasing maneuvers, where maneuvers are prescribed that cancel the differential $\Delta V$ of each CubeSat's deployment vector. The second method relies on artificial potential functions (APFs) to contain the spacecraft within a volumetric boundary and avoid collisions. Performance results and required $\Delta \mathrm{V}$ budgets are summarized, indicating that each method has advantages and drawbacks for particular applications. The mini phasing maneuvers are more predictable and sustainable. The APF approach provides a more responsive and distributed performance, but at considerable propellant cost. After considering current state of the art CubeSat propulsion systems, we conclude that the first approach is feasible, but the modified APF method of requires too much control authority to be enabled by current propulsion systems.
\end{abstract}

\section{Table of Contents}

1. INTRODUCTION $\ldots \ldots \ldots \ldots \ldots \ldots \ldots \ldots \ldots \ldots \ldots \ldots \ldots$

2. BACKGROUND $\ldots \ldots \ldots \ldots \ldots \ldots \ldots \ldots \ldots \ldots \ldots \ldots, 2$

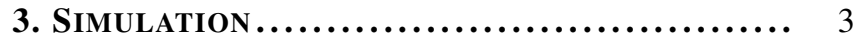

4. DEPLOYMENT DiSPERSION CONTROL......... 5

5. Artificial Potential function Method .. 7

6. Conclusions and Future Work ........... 10

ACKNOWLEDGMENTS ......................... 11

REFERENCES .............................. 11

BIOGRAPHY $\ldots \ldots \ldots \ldots \ldots \ldots \ldots \ldots \ldots \ldots \ldots \ldots \ldots, 11$

\section{INTRODUCTION}

Satellite swarms, as multiple spacecraft orbiting together, will enable novel science and technology missions. By providing spatially and temporally coordinated data collection, swarms

U.S. Government work not protected by U.S. copyright offer measurement capabilities not possible for single satellites or even constellations of spacecraft in separate orbits. We define spacecraft swarms as a formation of cooperative spacecraft in which the state of each spacecraft depends on the state of the other members in the formation. The scope of this discussion is limited to only swarms in Low-Earth Orbit (LEO). The intrinsic configuration of swarms in space faces major challenges related to the maintenance of their spatial disposition. Typically, each satellite in the swarm needs to perform maneuvers to keep the overall configuration, as well as to minimize the probability of collision. Since each satellite is in a distinct position and velocity state with respect to every other, differential motion acts as a threat to maintaining the swarm formation. Therefore, mission design requires a novel approach with respect to the traditional methods of uploading maneuvers with human involvement in the loop.

Our focus in this paper is a study of LEO swarm mission design by emphasizing the requirements for the intended application and the practical constraints of implementation and operations. An important consideration in swarm mission design is that the swarm configuration does not need to persist throughout the mission duration or for the entire orbit lifetime of the satellites. Swarm maintenance may be of relatively short timespan for collecting data into onboard storage. Then, the remaining mission duration addresses downlinking data in relatively brief contacts typically available from LEO. Atmospheric drag causes natural decay for the mission to meet disposal requirements within 25 years or sooner. After swarm maintenance ends, further data collection with less stringent temporal and spatial coordination is an option for certain mission concepts.

For this study, we model the relative satellite dynamics with NASA Ames' Swarm Orbital Dynamics Advisor (SODA), ${ }^{1}$ a simulation and analysis tool for satellites operating and maneuvering per a high level relative motion constraint. SODA simulates the dispersal of the deployed satellites to account for different orbit insertions that present a challenge to swarm maintenance. The simulation includes practical initial conditions, such as realistic deployment models, errors in the deployment vector, and delays between each spacecraft's deployment. This paper advances the research of the SODA tool by assessing control and maneuver strategies in the context of currently available CubeSat propulsion systems. 
We present results for two methods of maintaining swarm disposition. The first approach consists of small maneuvers that keep each spacecraft in a quasi-periodic range with respect to a reference point. This approach seeks to cancel out the main component of each spacecraft's deployment velocity vector via maneuvers that can be considered as miniature, modified phasing maneuvers. From these simulation results, propulsion systems are identified that would be suitable for implementation of this control scheme on CubeSats.

Next, we explored a method to achieve a random distribution of the spacecraft by defining a controller based on artificial potential functions (APFs). In this approach, spacecraft are contained within an ellipsoid volume constraint via an attractive potential, while repulsive potentials are assigned to each spacecraft to avoid collisions. Using position and velocity state information for all spacecraft in the swarm, maneuvers are calculated as a function of the time-varying APFs. To assess this method, we completed parametric studies for the selection of: volume container size, duty cycle of the impulsive maneuvers, maximum allowable $\Delta \mathrm{V}$ per maneuver, and the shape of repulsive function.

\section{BACKGROUND}

The concept of distributed functionality among smaller, less expensive spacecraft has been investigated for decades and is considered by NASA to be an enabling technology for future space missions. Practical implementation of spacecraft swarms relies on the precise control of relative distances and orientations between participating spacecraft. Researchers have considered various methods of achieving this by either finding orbits that together achieve a stable periodic relative motion, by utilizing a higher cost control effort to maintain formations in less ideal orbital environments, or a combination of the two.

By allowing instruments on separate spacecraft to be combined into a co-observatory, formation flying can replace an expensive multiple payload platform with a large number of low-cost spacecraft. ${ }^{3}$ Such cost-saving missions have consequently been of interest to NASA, ESA, and the DOD. One possible application is interferometric synthetic aperture radar (InSAR) in which a standard circular relative orbit that has periodic out of plane motion can used to create higher fidelity radar observations from Low Earth Orbit. ${ }^{6}$ Similarly, a ground-based laser communications to a chief spacecraft can be calibrated with the assistance of atmospheric probe lasers from deputy spacecraft to reduce power loss. ${ }^{5}$ Other examples include rapid stereographic imaging and other sparse antenna array applications.

Finding a formation that is at least partially facilitated by Keplerian motion, as was done for the Magnetic Multiscale Mission, ${ }^{7}$ while efficient from a control standpoint, involves a considerable orbit design effort and is potentially limited in the formation geometry that can be achieved. Methods of optimal formation trajectory planning can involve such methodologies as linear programming, primer vector theory, Hohmann transfers, Lambert's solution, and the application of Gauss' variation of parameters. ${ }^{6}$ A general method is to develop a set of optimal reference trajectories for a formation and then to use an LQR tracking method to control to each path. Alternatively, the desire to maintain any formation in a more demanding dynamical environment has led to the generation of a myriad of control methodologies applied to spacecraft formation configuration. However, a ground-based command and control system for swarm control for a large number of spacecraft would be insufficient to provide rapid corrective maneuver commands for swarm reconfiguration and collision avoidance in tight formations. Thus, the concept of autonomous formation flying has been of focus of this research. ${ }^{8}$

Scharf et.al. reduced the bulk of swarm control methods for various formations into five categorical architectures: Multiple-Input-Multiple-Output (MIMO), Leader/Follower systems in which individual spacecraft controllers are connected hierarchically, Virtual Structures in which spacecraft are treated as rigid bodies embedded in an overall virtual body, Cyclic systems where controllers are not hierarchical, and Behavioral systems which are a combination of one or more of the previous systems. ${ }^{3}$ The advantages and disadvantages of MIMO systems are well known. Although such systems are optimal and stable, they demand a massive amount of inter-spacecraft communication and data transfer to operate and are not robust against local system failures, such as a single thruster failure. The Leader/Follower approach reduces the swarm control problem to one in which each spacecraft must simply follow at least one other, thereby reducing the amount of information needed by each controller to track its target. However, this approach reduces the state information requirement at the cost of optimality. Cyclic algorithms are non-hierarchical control strategies and encompass all Virtual Structure systems. The artificial potential function control method is one example of a cyclic system. Stability of these systems is difficult to prove analytically and thus is generally studied through simulation. Behavioral systems are widely seen in robotics and are simply described as a combination of any of the preceding formation control architectures.

The Clohessy-Wiltshire model of satellite relative motion is ubiquitous in the area of formation flight and nearly always used as the initial step of any satellite swarm control law derivation. The linearized equations of motion have, most notably, been used to calculate the optimal set of impulsive maneuvers to bring a deputy spacecraft to a target state (usually the origin of the system). ${ }^{4}$ Vassar et. al utilized discrete Clohessy-Wiltshire state equations to develop closed-loop optimal control laws for maintaining a formation between a pair of satellites in a circular orbit. This work demonstrated that in-plane motion required much more frequent correction than out-of-plane motion. ${ }^{5}$ Redding et. al. built off of Vassar's design in creating a linear quadratic station-keeping controller as a potential autopilot mode for the STS Orbiter. This system consisted of a Linear Quadratic Regulator (LQR) feed-back controller that expands on Vassar's work and a feed-forward controller used when the relative state error is above a given threshold. The latter controller computes larger corrective maneuvers as a function of the relative state error and the steady-state feed-forward control that follows directly from the Clohessy-Wiltshire model. ${ }^{9}$

Lopez and McInnes introduced artificial potential functions as a control method by defining the local topology around a spacecraft and imbedding that into a Lyapunov potential function. This topology generates analytical guidance commands obtained using Lyapunov's second method. The guidance algorithm ensures convergence on a target along the path of steepest descent in the potential field. Additionally, a spacecraft is steered clear of possible collisions by superimposing high potential fields around nearby objects such as 
other spacecraft. ${ }^{2}$ Conn et. al. adapted this terminal guidance method to instead contain a swarm of free-moving satellites within a given ellipsoidal volume. ${ }^{1}$

\section{Simulation}

\section{Deployment}

SODA includes two types of deployment simulations that we term VNC directional and rotational. The VNC directional deployment dispenses the CubeSat in the direction of one of the deployer's velocity-normal-conormal (VNC) axes. The xaxis is aligned with the velocity vector, the y-axis is aligned with the orbit normal, and the $\mathrm{z}$-axis completes the orthogonal triad. Deployment along these axes correspond to in-track, cross-track, and radial deployments, respectively. Figure 1 illustrates this configuration for a normal directional deployment.

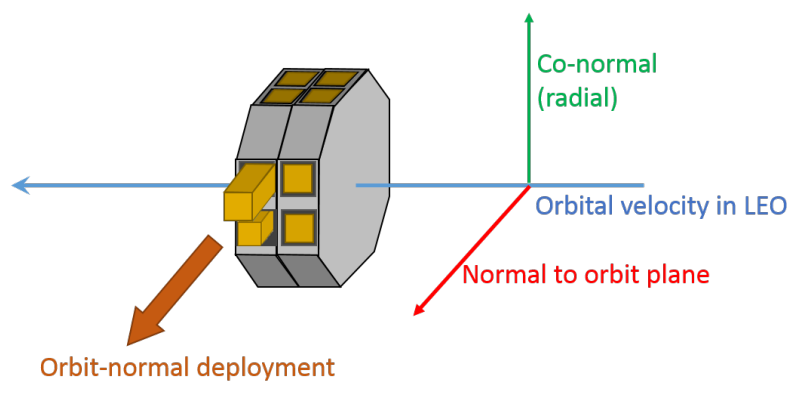

Figure 1. Depiction of a directional deployment in the normal axis of the VNC coordinate frame.

The second deployment type simulates CubeSats launched on a vehicle that is rotating $6 \mathrm{deg} / \mathrm{sec}$ about the velocity vector, with deployers positioned at $30 \mathrm{deg}$ with respect to the velocity vector. For this rotational deployer, satellites are jettisoned with large differences in all velocity vector components since the rotation imparts a $\Delta \mathrm{V}$ in the $\mathrm{Y}-\mathrm{Z}$ plane. Figure 2 illustrates this scenario.

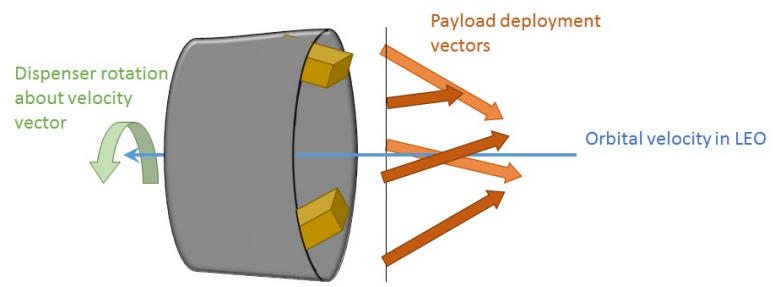

Figure 2. Depiction of a rotational deployment. The vehicle's rotation introduces random components in the Y-Z plane each time a deployment is simulated.

In both simulation cases, there is a two second delay between the dispensing of each CubeSat. We model the typical mechanism for CubeSat deployment, a spring force. Because the force vector is slightly variable deployment to deployment, each CubeSat's simulated deployment force includes perturbations. For a $14 \mathrm{~kg}$ satellite, the $\Delta \mathrm{V}$ magnitude was sampled from a distribution with mean $1.2 \mathrm{~m} / \mathrm{s}$ and $3 \sigma=10 \%$.

Pointing errors are included by applying a rotation around the unit vector of the intended deployment vector. Each iteration, the vector is rotated about an arbitrary axis by an angle sampled from a Gaussian distribution (mean $=0$, $3 \sigma=0.5 \mathrm{deg}$ ). Equation 1 is the applied rotation matrix, ${ }^{16}$ where $\mathbf{u}$ corresponds to the unit vector of the arbitrary axis used for the rotation, $\otimes$ is the tensor product, $\mathbf{I}$ is the identity matrix, $\mathbf{u}_{\times}$is the cross product matrix of the unit vector, and $\phi$ is the angle of rotation.

$$
\mathbf{R}=\cos \phi \mathbf{I}+\sin \phi[\mathbf{u}]_{\times}+(\mathbf{1}-\cos \phi) \mathbf{u} \otimes \mathbf{u}
$$

We illustrate the effect of deployment direction in Figure 3, which summarizes the results of 1000 Monte Carlo style simulations for each of the deployment types described above. No control was applied; the spacecraft were in free drift for one day. Each histogram subplot shows the number of cases when the deployed satellite reaches a given separation distance from the deployer. For deployments in the velocity vector direction, greater separations are produced since the applied $\Delta \mathrm{V}$ is mainly in the direction of orbital motion. Normal and co-normal deployments result in about the same separations. The rotational deployment type produces greater dispersion due to the spacecraft being ejected into different orbital planes, namely slightly different inclinations.
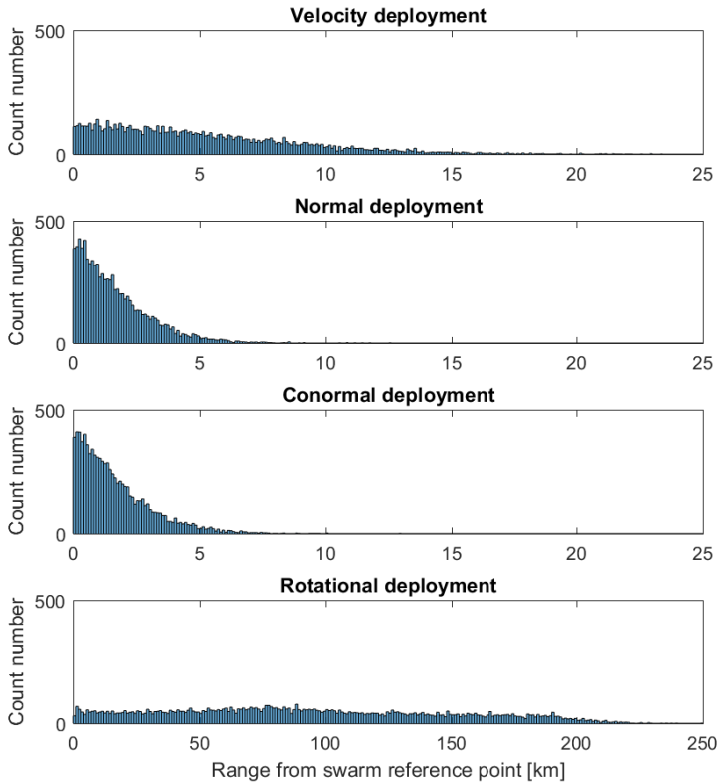

Figure 3. Simulations of uncontrolled motion demonstrate that deployments in the velocity vector direction or from a rotational deployer result in the largest separations.

These free drift simulations emphasize the importance of considering the effects of swarm deployment design in the overall mission scenario. Rotational and velocity vector deployments will cause greater dispersion, requiring control cost early in the mission to ensure swarm containment. Conormal and normal deployments are more advantageous choices in that regard; however, even deployments in these directions will suffer from pointing inaccuracy and may also require some corrective maneuvers to counteract any deployment compo- 
nent in the velocity vector direction. Of course, the goal is not to avoid dispersion entirely, since some separation is required to avoid risk of collision. Thus, swarm deployment and controller design becomes an exercise in simultaneously containing the spacecraft, but preventing collision.

\section{Volume container swarm type}

A volume container swarm type takes the form of an ellipsoid boundary constraint on the satellites' motion. The center of the ellipsoid is defined via a state vector and has its own virtual orbit. Spacecraft are prescribed maneuvers to remain inside this volume while avoiding collisions with other spacecraft. Let the parameters $a, b$, and $c$ specify the dimensions of the volume container, as in:

$$
\frac{x^{2}}{a^{2}}+\frac{y^{2}}{b^{2}}+\frac{z^{2}}{c^{2}}=1
$$

where $a, b$, and $c$ define the dimensions of an ellipsoid in the $\mathrm{x}-, \mathrm{y}-$, and $\mathrm{z}$-axis directions of the local-vertical, localhorizontal (LVLH) frame, with origin at the center of the volume container. In other words, for a local frame with its origin on the user-specified orbit, $a$ would be the dimension in the radial direction, $c$ would be the dimension in the orbit normal direction, and $y$ would be in the direction of the unit vector that completes the triad. For simplicity, we studied the case of a spheroid, where the constants, $a, b$, and $c$ were equal for several spheroid sizes.

For the simulations in this study, the volume center point was defined as follows. Following the simulated deployment of all spacecraft, the spacecraft closest to the swarm centroid is identified. Its state vector is defined as the LVLH origin and is propagated in time, subjected to a drag area equal to the average of the drag areas of all spacecraft at the initial state epoch. Taking this approach results in the swarm decaying in altitude together. Note that the spacecraft closest to the initial centroid would usually not expend any fuel. This consequence may be exploited by rotating the designated volume center point amongst various spacecraft, or assigning this role to the spacecraft lowest on fuel reserves.

An alternative method of defining the volume center it to explicitly define the center point in terms of orbital elements. In the case of LEO swarms, when this reference point is propagated as a point mass, it is not subjected to the drag forces that the swarm satellites experience. As atmospheric drag causes the spacecraft to decay in altitude, the volume center remains on a non-decaying orbital path and essentially serves as a stationkeeping reference point. Maneuvers are prescribed that raise the altitude of the spacecraft to remain within the volume boundary. For particular science requirements, this approach may be desirable, but it has a higher fuel cost.

\section{Spacecraft}

Typical 1 to 6 unit CubeSats range from $1-14 \mathrm{~kg}$ mass. Their capabilities vary depending on the available volume. In this study, we selected the form factor of a $6 \mathrm{U}$ CubeSat with $14 \mathrm{~kg}$ of mass, which is small enough to enable scalability to large swarms and sufficiently large to accommodate valid science payloads and propulsion systems. $6 \mathrm{U}$ CubeSats have extensively flown in space, performing several successful missions.

Our simulations assume that ten CubeSats launch together and are inserted into a nearly-circular Low Earth Orbit (LEO) with an altitude of $500 \mathrm{~km}$. We focus our analysis on studying the differential motion created by the dispersion in
Table 1. Properties of common propulsion systems.

\begin{tabular}{|c|c|c|}
\hline System & Isp (s) & Thrust \\
\hline \hline Cold gas & $30-65$ & $1-50 \mathrm{mN}$ \\
Non-toxic monopropellant & $200-240$ & $0.1-1.25 \mathrm{~N}$ \\
Bipropellant & $300-320$ & $1-2 \mathrm{~N}$ \\
Electrospray/FEEP & $1500-5000$ & $0.01-1.5 \mathrm{mN}$ \\
Pulsed Plasma Thrusters & $1500-3000$ & $1-300 \mu \mathrm{N}$ \\
\hline
\end{tabular}

the deployment and therefore, we consider all satellites to have a constant and identical ballistic coefficient, avoiding differential drag dispersion effects. A constant drag area of $280 \mathrm{~cm}^{2}$ was defined for each satellite.

Note that our scope is mainly limited to the propulsion subsystem performance and does not address other subsystem requirements. For example, we assume state estimation and communication capabilities that enabled perfect sharing of spacecraft position and velocity vectors.

\section{Propulsion Subsystem}

There are several technologies that provide propulsion for CubeSats. Some commercial off-the-shelf (COTS) units have successfully operated in space, most being cold gas systems. Industry is moving towards non-toxic monopropellant and electric propulsion systems that can provide higher $\Delta \mathrm{V}$ capability for the same amount of propellant mass due to higher specific impulses. Table 1 summarizes propulsive technologies available for CubeSats and their performance characteristics.

Cold gas systems are traditionally a reliable and simple solution for several mission concepts. VACCO Inc. has extensive experience with cold gas systems, including self-contained units like the NASA C-POD and CuSP micropropulsion systems that have low power, $25 \mathrm{mN}$ cold gas thrusters. They also provide custom designs for interplanetary missions, such as the JPL MarCO micropropulsion system, that has four axial and four RCS cold gas thrusters, providing $755 \mathrm{Ns}$ of total impulse. These systems are compatible with the R-134a and R-236fa refrigerants as propellants, enabling specific impulses between 40-60 seconds. ${ }^{11}$ Alternatively, Gomspace has developed a cold gas system that is at a technology readiness level (TRL) 9 after being demonstrated on orbit in a $3 \mathrm{U}$ CubeSat. A larger version is planned to fly in a $6 \mathrm{U}$ mission in 2018. The Gomspace NanoProp $6 \mathrm{U}$ propulsion system has four nozzles that can provide thrust up to $10 \mathrm{mN}$ and a specific impulse between $60-110$ seconds. ${ }^{12}$

An alternative to cold gas systems are non-toxic monopropellant propulsion systems that are the preferred technology for large $\Delta \mathrm{V}$ maneuvers. Most non-toxic systems utilize either AF-315E (HAN) or LMP-103S (ADN) propellants that provide higher performance than traditional hydrazine in terms of specific impulse and density. Both of these propellants enable specific impulses in the range of 200-250 seconds. VACCO produces LMP-103S propulsion systems in collaboration with Bradford ECAPS. ${ }^{11}$ The $6 \mathrm{U}$ Lunar Flashlight mission incorporates an ADN propulsion system that uses four $100 \mathrm{mN}$ thrusters, providing $237 \mathrm{~m} / \mathrm{s}$ for a $14 \mathrm{~kg}$ spacecraft. Bradford ECAPS also developed $1 \mathrm{~N}$ thrusters that have flown as part of the PRISMA and Skysat 3-7 missions. ${ }^{15}$ 
Bipropellant systems are also in development for small satellites. These systems have the advantage of providing specific impulses of 300-320 seconds, higher than traditional monopropellant systems. As an example, the HYDROS-C thruster uses water as propellant and can provide thrust at $1.2-1.75 \mathrm{~N}$, with a specific impulse of 310 seconds. ${ }^{10}$

Electric propulsion systems are another option for the constrained volume and mass allowances of CubeSats. They provide significantly higher specific impulse than their chemical counterparts, at a trade for power and thrust magnitude. These systems are ideal candidates for swarm concepts where maneuvers may be lower in thrust but performed more frequently. Accion Systems Inc. ${ }^{14}$ and Enpulsion ${ }^{13}$ are developing electrosprays and field emission propulsion systems, that provide an interesting compromise between power consumption and thrust. These systems can be throttleable and have neither moving parts nor complex propellant feed systems. The specific impulse can be configured to be 1500 to 5000 seconds, while the thrust magnitude is in the range of $\mu \mathrm{N}$ to $\mathrm{mN}$. Other systems in development include Hall effect thrusters and ion engines, but the power required for these technologies requires larger satellite form factors.

\section{Trade Space Analysis}

SODA enables a trade space study in which propulsion systems and deployment strategies are evaluated in the context of swarm missions. Maneuvers are computed based on nondeterministic conditions, since the evolution of each swarm is a function of the deployment and the differential motion between the satellites. Therefore, the swarm has to manage itself to maintain its configuration within the volume limits and prevent collision. To study the effects of volumetric boundary size and $\Delta \mathrm{V}$ capabilities, we did not vary other spacecraft parameters such as volume and mass properties, as defined in the Spacecraft subsection.

Other variables and characteristics fixed for all simulations include a nearly circular (0.001 eccentricity) orbit with $500 \mathrm{~km}$ altitude, a full Earth gravity field, and an atmospheric drag model. For the control algorithms, we used a representative initial state vector from each deployment type to compare the effects deployment direction. Ultimately, the goal is to identify trends in propulsion system characteristics and controller design parameters that yield successful performance for various spheroid sizes.

\section{DEPLOYMENT Dispersion CONTROL}

\section{Method}

In general, deployment dispersion control requires few and small maneuvers. For certain scenarios, even a single initial adjustment maneuver will suffice, i.e. short duration missions when no differential drag is involved. The differences in the deployment vectors cause the satellites to drift apart from one another. In the VNC reference frame, the factor that contributes most to the swarm drift is the component of the deployment vector that is aligned with the orbital velocity. The deployment dispersion control consists of a maneuver to cancel out the difference in the velocity component between the reference point of the swarm and each of the members. The maneuver that accomplishes the cancellation occurs only along the orbital velocity vector. Accurate and timely knowledge of the deployment vectors could enable the implementation of the algorithm by just cancelling the velocity component in the VNC reference frame. However, the required precision for the measurement of the spring force and attitude control of the deployer may not be achieveable with current satellite operations and technology. The deployment dispersion control method depends then on the shared knowledge of the state vectors among satellites, so the computer on board each satellite can negate the differences in semimajor axis between it and the considered reference point. For our analysis, the reference point of the swarm corresponds to satellite number 5, (equivalent to half of the total number of swarm members). However, the deployment dispersion control is robust and works with any satellite or moving orbital point as a reference.

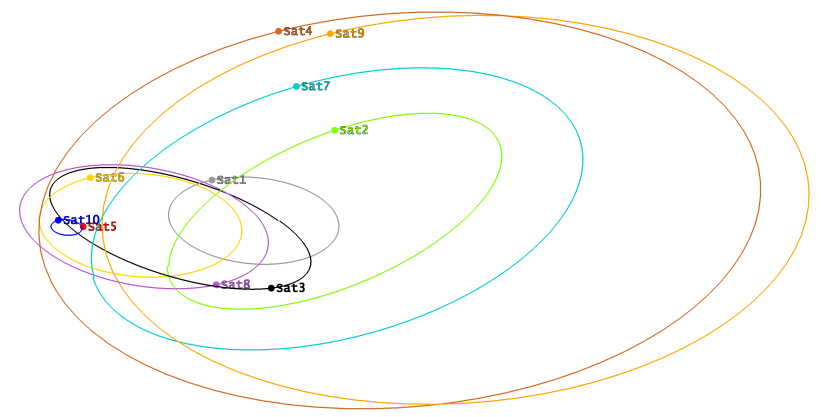

Figure 4. Spacecraft motion relative to satellite 5 (shown in red), the reference point of the swarm. Shown are the stable and quasi-periodic trajectories resulting from a rotational deployment and applied control maneuvers.

Since the perturbations introduced by the deployment are distinct every time the simulation executes, each satellite will drift apart from the reference point at a different rate. Similarly, depending on the type of deployment the other two components, normal and co-normal, will determine the type of motion with respect to the reference point as well. Differences in these two components can imply larger periodic oscillations in the planes defined by these two directions, i.e., different relative orbit tracks with respect to the reference point. These tracks can have various relative inclinations and sizes as in Figure 4.

The initial maneuver to stop the drift over time and instead, to stay in a quasi-periodic track, can be timed to allow a safe inter-satellite range that satisfies particular mission requirements. Since the deployment vector is different for each of the satellites, they will drift at various rates. The mission design can determine then when to place a maneuver to get a quasi-periodic track with no risk of collision since the tracks can be at ranges of hundreds of meters or kilometers, depending on the mission concept. Therefore, each satellite can measure its drift rate from the reference point and perform a maneuver that cancels the differential deployment in the velocity component to stop the continuous drift and to instead stay at a particular variable range for several days, in the absence of differential drag.

\section{Results}

We used the three directional and the rotational deployments described in the Deployment subsection. For any scenario, the method showed robustness to the deployment, with the only difference of the magnitude of the necessary maneuver to stop drifting as result of the deployment vectors. Results for a test case of each scenario appear in Figure 5.

In-track direction deployments require higher $\Delta \mathrm{V}$ magni- 

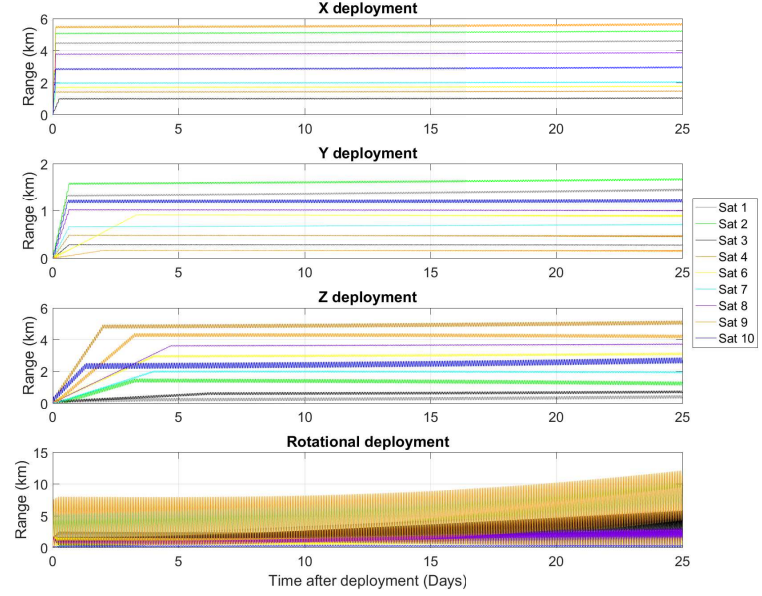

Figure 5. For each of the representative deployment scenarios, maneuvers were performed to control the drift of each spacecraft away from the swarm reference point. The resulting relative motion is an oscillation whose amplitude depends on the $y$ - and $z$-axis components of the deployment vectors.
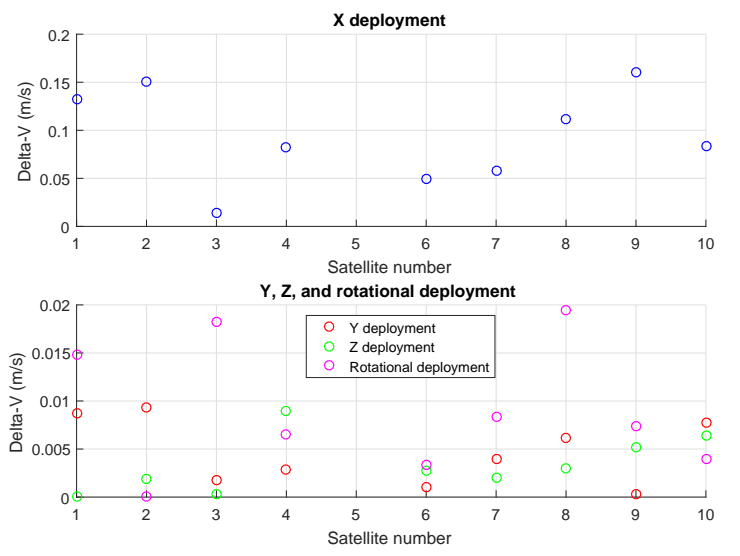

Figure 6. $\Delta V$ cost per satellite for each deployment scenario. In this case, satellite 5 does not perform corrective maneuvers since it serves as the swarm's reference point.

tudes for the initial maneuver of each satellite since the differential component of the deployment vector that is aligned with the velocity vector of the spacecraft is higher in those cases. Since our nominal deployment is $1.2 \mathrm{~m} / \mathrm{s}$ and we allow a difference of $10 \%$ in the magnitude and $0.5 \mathrm{deg}$ in the pointing, the required $\Delta \mathrm{V}$ for the in-track deployment is on the order of $0.1 \mathrm{~m} / \mathrm{s}$ as expected. The maneuvers are on average an order of magnitude smaller for the rest of the deployment scenarios. For normal and conormal deployments, the maneuvers place each satellite in a quasi periodic state for as long as 25 days as shown in Figure 5.

For rotational deployments, the Y-Z components have higher magnitudes than with the directional scenarios. That causes the satellites to drift farther apart over time and to have in most cases higher amplitudes as shown in Figure 5, where satellite 9 has an increasing range with an amplitude of a few kilometers. However, in the same scenario we can also find small amplitudes and quasi-stable periodic motion as in the directional deployments. That is the case of satellite 10 in this representative simulation that stays in a small track of just under $1 \mathrm{~km}$ for a long period. The differences in the relative tracks with one another can be seen in Figure 4, which corresponds to the rotational case described in this section. The higher dispersion the rotational deployment imparts to the satellites implies that either the mission has to consider a more controlled deployment strategy, or more maneuvers need to be performed with another strategy that can control out of plane motion.

\section{Discussion}

Deployment control results - The results demonstrate the applicability of the deployment correction method to swarm control. For this analysis, we considered the same initial state vectors as in the APF approach. The deployment control method first considers the differences in free drift among satellites, seeing that distinct $\Delta \mathrm{Vs}$ imparted at deployment resulted in various separation rates. The method uses maneuvers timed at multiples of the reference orbital period to counteract the initial relative drift.

The swarm size was not in this case a hard constraint; we set our initial goal to keep the swarm at a maximum of 15 $\mathrm{km}$. However, for the Y (or normal) direction, the differential velocity did not impose a high separation rate and we accomplished a small spheroid swarm (under $2 \mathrm{~km}$ ).

The outcome of each simulation gave 3-D motion in the VNC system of the reference point, therefore the satellites wander in the speheroid swarm type. In this control method, the motion of each member is more predictable than for the APF implementation: after the initial maneuver, they enter a quasiperiodic trajectory in the reference coordinate system that keeps them with a stable range. The separation distance varies depending on the position along track, which is perturbed over long term propagation and degrades. For the study of directional deployment cases with 25 day propagations, the satellites did not present large degradations of the relative distance over time. Some of them experience slight changes in their range at the end of the simulation, suggesting that maintenance maneuvers could be included in extended mission durations.

The maneuvers for these examples were timed to control the relative ranges. Maneuver timing was selected by design for control of both the relative inter-satellite range and the threat of collision. The swarm can be managed to accommodate mission requirements for larger or smaller inter-satellite distances.

In addition to maneuvers, swarm control utilizes relative motion. Since the satellites have distinct relative inclinations in the reference point coordinate system, swarm member trajectories can avoid or minimize collisions. Even if their ranges to the center are similar, the relative distances between them can be large, since they travel in their own relative tracks with low probability of collision.

Realistic swarm scenarios will involve corrections over time. Example perturbations are differential drag or large deployment dispersions. The rotational deployment case gave an example of the latter scenario, where the $\mathrm{Y}$ and $\mathrm{Z}$ components of the deployment vectors for satellite 9 and for satellite 4 
with respect to the reference, satellite 5, are higher than the rest. The differences appear in Figure 4 and Figure 5. As a consequence, the relative trajectories or tracks for those two members have larger dimensions and their motion is also less stable, which would require a potential maintenance maneuver for long term missions. The same figures also show the opposite effect in other members; by the nature of the modeled rotational deployment, smaller differential magnitudes in the $\mathrm{Y}$ and $\mathrm{Z}$ components of the deployment vectors are also feasible. Satellite 1, 5, or 10, are great examples since their separation rate was slower and also the $\mathrm{Y}$ and $\mathrm{Z}$ differential magnitudes were small, resulting in relative tracks that are close to the reference point and also small in size, which translates into more stable oscillations in the amplitude of the quasi-periodic range.

Propulsion solutions for the deployment control approachThe classic approach provides promising results from a mission implementation perspective. Even though the method is more predictable and the satellites pass through very similar tracks over time, which may be or not be in agreement with the desired requirements of a mission, the affordability of this scenario makes it very attractive. The propellant budget that is necessary for the deployment control approach is very modest and therefore a wide range of existing propulsion systems have capability to meet the total impulse required.

As an example, cold gas systems provide the smallest specific impulses of all the considered propulsion technologies in this paper, yet they are capable of supporting the worst of our simulated cases. For a scenario where only an initial maneuver is needed, the worst case of a directional deployment in the velocity direction corresponds with satellite 9 and would result in a required maneuver of $0.16 \mathrm{~m} / \mathrm{s}$, as shown in Figure 6. Satellite 9 would need a propellant mass budget of $5 \mathrm{~g}$ of cold gas. Tanks that store propellant masses between $10 \mathrm{~g}$ to $1 \mathrm{~kg}$ are in line with current state of the art CubeSat propulsion systems. Moreover, the thrust magnitude needed to accomplish the $\Delta \mathrm{V}$ of the initial maneuver, and any additional maintenance maneuvers, is sufficiently small to be accomplished with majority of the chemical and even electric propulsion systems represented in the survey.

The order of magnitude required for the impulsive maneuvers is in the $\mathrm{mm} / \mathrm{s}$ to $\mathrm{m} / \mathrm{s}$ range, so the minimum impulse bit provided by the propulsion system has to be small. For $6 \mathrm{U}$ CubeSats with $12-14 \mathrm{~kg}$ of mass, most typical electric systems would provide minimum impulses of $10 \mathrm{e}-7$ to $10 \mathrm{e}-5 \mathrm{~m} / \mathrm{s}$ $\Delta \mathrm{V}$. Typical chemical systems would give minimum impulse bits $10 \mathrm{e}-1$ to $10 \mathrm{e}-3 \mathrm{~m} / \mathrm{s}$ range for bipropellant systems, $10 \mathrm{e}-6$ to $10 \mathrm{e}-4 \mathrm{~m} / \mathrm{s}$ for cold gas, and $10 \mathrm{e}-4$ to $10 \mathrm{e}-2 \mathrm{~m} / \mathrm{s}$ for non toxic propulsion systems. High precision per maneuver is required for accurate insertion into the quasi-periodic range relative track. Thrust values provided in Table 1 correspond to typical nominal performance. Most of the systems are throttleable and would provide enough thrust precision to achieve the distinct $\Delta \mathrm{V}$ magnitudes for the variations in deployment. For electric systems, the achievable maximum thrust would depend on the amount of power available for propulsion. However, if we avoid the impulsive maneuver assumption, and present the alternative of finite burns, the amount of $\Delta \mathrm{V}$ required can be accomplished by firing the electric propulsion system for a longer time.

\section{Artificial Potential function METHOD}

\section{Method}

We described SODA's implementation of APFs in a previous manuscript, ${ }^{1}$ and we briefly summarize the method here. Containing the swarm within a defined boundary is accomplished via an attractive potential function, while avoiding spacecraft collisions is accomplished by repulsive potential functions. The attractive potential function is given by:

$$
\phi_{a, i}=\frac{1}{2} \bar{r}_{i}^{T} P \bar{r}_{i}
$$

where $\bar{r}_{i}$ is the position of the spacecraft in the LVLH frame and $P$ is a $3 \times 3$ positive-definite matrix that describes the shape of the attractive potential function. The repulsive potential function is given by:

$$
\phi_{r, i}=\sum_{j=1}^{n, j \neq i} A e^{-B\left(\bar{\rho}_{j, i}^{T} P \bar{\rho}_{j, i}\right)}
$$

where $\bar{\rho}_{j, i}$ is the position of satellite $j$ with respect to satellite $i$. Tuning the constants $A$ and $B$ in Equation 4 is very important; modifying $A$ and $B$ modifies the overall swarm behavior. For example, choosing values for a more conservative repulsive potential function will result in greater fuel use, as SODA will seek to maintain larger intersatellite distances. The total potential is given by:

$$
\phi_{i}=\phi_{a, i}+\phi_{r, i}
$$

As the mission simulation progresses, if the time derivative of an individual satellite's potential is negative, it performs no maneuvers. Otherwise, SODA queries the states of all satellites in the swarm at a user-specified frequency, and prescribes a relative velocity change for each spacecraft.

The simulations propagated all satellites for one day, allowing sufficient time to determine trends in the $\Delta \mathrm{V}$ requirements as the satellites spread. Simulations took into account all combinations possible from the parameters listed in Table 2. For the repulsive potential functions, we studied the effects of changing the $A$ and $B$ parameters.

Table 2. Variable parameters in the APF simulations.

\begin{tabular}{|l|l|}
\hline Parameter & Values \\
\hline \hline Spheroid radius $(\mathrm{km})$ & $0.1,0.5,1,5$ \\
Duty cycle $(\mathrm{s})$ & $1,10,30,60$ \\
A, B & $50,100,500,1000,3000,5000$, \\
& 7000 \\
Max. $\Delta v(\mathrm{~m} / \mathrm{s})$ & $1 \mathrm{e}-4,1 \mathrm{e}-3,1 \mathrm{e}-2,1 \mathrm{e}-1,1$ \\
Min. $\Delta \mathrm{v}(\mathrm{m} / \mathrm{s})$ & $1 \mathrm{e}-7,1 \mathrm{e}-6,1 \mathrm{e}-5,1 \mathrm{e}-4,1 \mathrm{e}-3$ \\
Deployment & Rotational, Directional (VNC) \\
\hline
\end{tabular}


To identify sustainable swarm scenarios and successful configurations, we defined success criterion as: maximum distance of any satellite from the center of the spheroid, $\Delta \mathrm{V}$ spent in a one day simulation, and the minimum distance between any satellite pair (only assessed later than 20 minutes after deployment). The last parameter quantifies the performance of the repulsive potential function preventing collisions. The success criterion for swarm maintenance was that no satellite move away from the volume center by more than $200 \%$ of the spheroid radius. The maximum $\Delta \mathrm{V}$ budget for our $6 \mathrm{U}$ form factor was: $400 \mathrm{~m} / \mathrm{s}$ for high $\Delta \mathrm{V}$ cases of more than $1 \mathrm{e}-4 \mathrm{~m} / \mathrm{s}$, and $800 \mathrm{~m} / \mathrm{s}$ for low $\Delta \mathrm{V}$ cases of less than $1 \mathrm{e}-4 \mathrm{~m} / \mathrm{s}$ (where we assume electric propulsion systems with high specific impulses). We allowed a minimum 5 meters inter-satellite range; values less than this are considered failures due to collision.

\section{Results}

Simulation results identify an area in the trade space that satisfies the defined success criteria: maneuvers with maximum allowable $\Delta \mathrm{V}$ of $1 \mathrm{~m} / \mathrm{s}$, a desired spheroid with $1 \mathrm{~km}$ radius, and a duty cycle of 10 and $30 \mathrm{~s}$. Figures 7, 8, and 9 show the results of simulations of the rotational deployment with a maximum allowable $\Delta V$ magnitude of $1 \mathrm{~m} / \mathrm{s}$. In Figure 7 , the markers shown in orange correspond to the successful cases, purple markers indicate cases that failed one or more success criteria. Figure 8 shows the same test cases but with a color map indicating the maximum separation distance from the center of the volume container over one day. Cases with separation greater than $5 \mathrm{~km}$, shown in yellow, are scenarios where the swarm was not contained at all, with maximum separation of hundreds or even thousands of kilometers. Figure 9 shows results for a rotational deployment and a maximum burn size of $0.1 \mathrm{~m} / \mathrm{s}$. In these test cases, no combination of possible configuration parameters resulted in a successful case per our success criteria. Moreover, all simulations spread significantly, showing that a burn size of this magnitude cannot compensate differential motion over the timespan of a day. Further analysis should include smaller step in the maximum allowable burn size to refine the available solution space. For example, a burn size of $0.5 \mathrm{~m} / \mathrm{s}$ may open the solution space.

Figures 10, 11, and 12 show how tuning the repulsive potential function parameters $A$ and $B$ affects swarm performance in terms of total $\Delta \mathrm{V}$ required, the maximum separation distance from the center of the volume container, and the minimum separation distance between satellites. Simulations represented have a duration of one day, a duty cycle of $30 \mathrm{~s}$ and a desired spheroid radius of $1 \mathrm{~km}$. In the parametric studies of each of the four deployment scenarios, the same initial conditions were applied while other parameters were varied. From these test cases, only $A$ and $B$ values greater than or equal to 500 met the success criteria for at least one of the deployment types. While the process of tuning the APF parameters warrants further refinement, it is clear that these variables should be evaluated in the context of overall mission goals. For example, the $A$ and $B$ values of 3000 produce low $\Delta \mathrm{V}$ and tight swarm control for deployment in the normal direction.

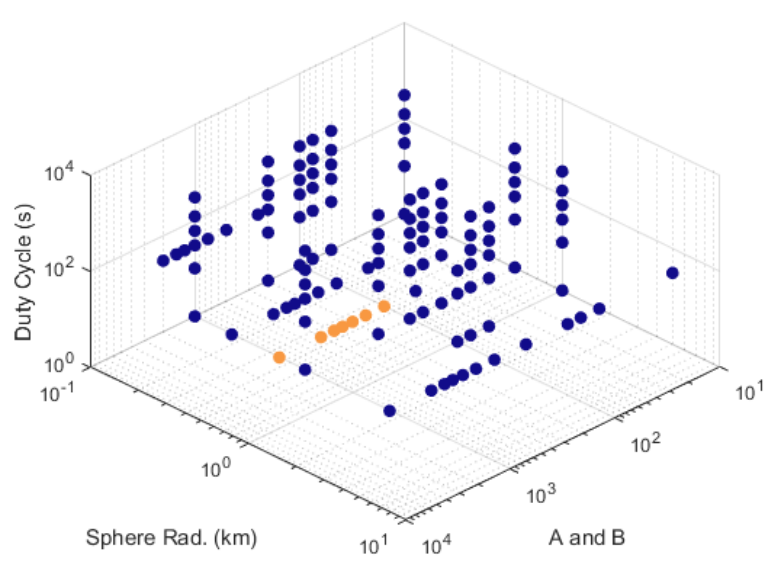

Figure 7. Simulations of rotational deployment revealed some cases that met the success criteria, shown in orange.

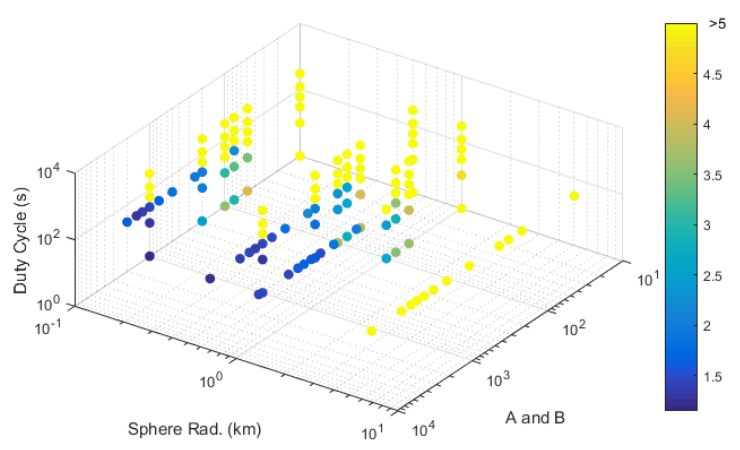

Figure 8. This figure shows the same parametric study results as Figure 7, with the markers' color indicating maximum excursion (in $\mathrm{km}$ ) of any spacecraft from the swarm center.

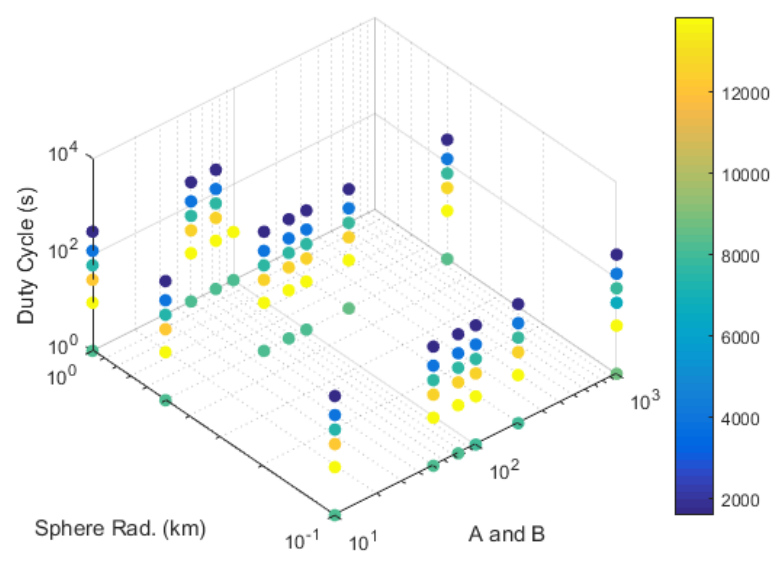

Figure 9. For parametric study rotational deployments with $\Delta V ; 0.1 \mathrm{~m} / \mathrm{s}$, maximum separations (units of $\mathbf{k m}$ ) indicate uncontained relative motion in all cases. 


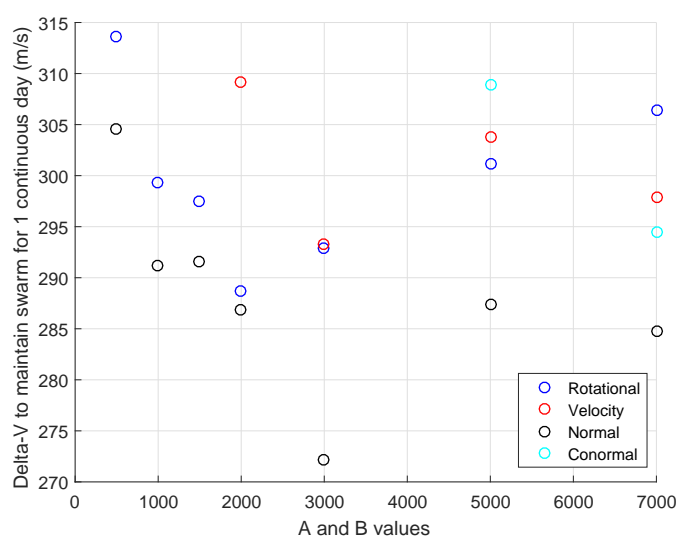

Figure 10. The $\Delta V$ totals required for swarm maintenance are shown for all test cases that met success criteria identified in the parametric studies.

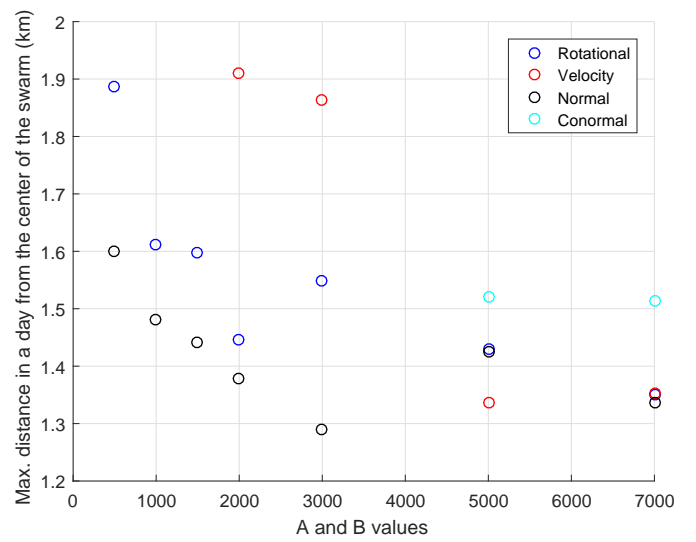

Figure 11. The maximum distances of any spacecraft from the volume container center are shown.

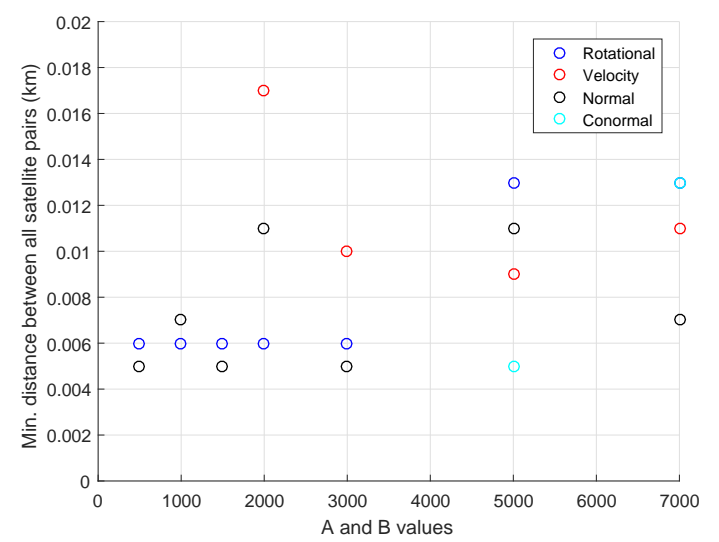

Figure 12. Minimum intersatellite distances are shown, indicating how close the spacecraft came to collision.
To further examine the swarm performance, we assessed a particular configuration from the set of successful cases: a rotational deployment case with 30 seconds duty cycle, maximum allowed $\Delta \mathrm{V}$ of $1 \mathrm{~m} / \mathrm{s}$, and $A=B=5000$. This simulation meets the success criteria with a $\Delta \mathrm{V}$ budget of $301 \mathrm{~m} / \mathrm{s}$, maximum distance from the center of $1.43 \mathrm{~km}$, and minimum separation of 13 meters. Figures 13 and 14 show this successful case that maintains a swarm within $200 \%$ of the desired spheroid radius, while avoiding a risk of collision.

Figure 13 demonstrates that maneuvers are prescribed that keep the swarm together when any of the units are found outside the spheroid boundary. Excursions outside the spheroid are controlled by maneuvers after the duty cycle is met. The average distance from the center of the spheroid is within the desired size.

Figure 14 summarizes the separation between all satellites in the swarm, illustrating that closest approaches are above our recontact avoidance requirement of $15 \mathrm{~m}$. Note that a duty cycle of 30 seconds has the consequence of preventing satellites from immediately performing a maneuver when required, even if the separation constraint is met. Also, high $A$ and $B$ values allow the satellite repulsive potential functions to be distinguishable in the overall potential field for small spheroid sizes. The algorithm works correctly by sensing the spacecraft at a safe distance for this dimension, avoiding collisions.

\section{Discussion}

APF results-By analyzing test cases that met success criteria, we can identify two lessons learned for swarm design. First, the more that the spacecraft spread, the higher the $\Delta \mathrm{V}$ required to compensate for the drift and to bring the swarm back together. This effect can be avoided by performing maneuvers prior to breaching volumetric boundary. We also note that reducing the propulsion system duty cycle would allow more frequent maneuvers, allowing the control system to return the spacecraft to the swarm more quickly. Of course, this would come at the cost of propellant. From the duty cycles included in this parametric study, $30 \mathrm{~s}$ was a sufficient compromise between propellant mass consumption and swarm maintenance.

Second, the more conservative the collision avoidance maneuvers are, the more propellant is spent. Maneuvers are not only required to avoid collisions, but also to correct the resulting trajectory. For example, if a maneuver is performed to avoid a satellite within the swarm, and that maneuver is directed in a different direction than the natural coasting orbital motion of the satellite, the satellite likely will escape the swarm as a consequence of that avoidance maneuver. Thus, the satellite must spend propellant in the initial maneuver and subsequently to return to the swarm. The repulsive functions that regulate this behavior must be tuned accordingly for the swarm volume size. Arbitrary choices for the exponential functions defining the repulsive and attractive potential functions will not be successful. 


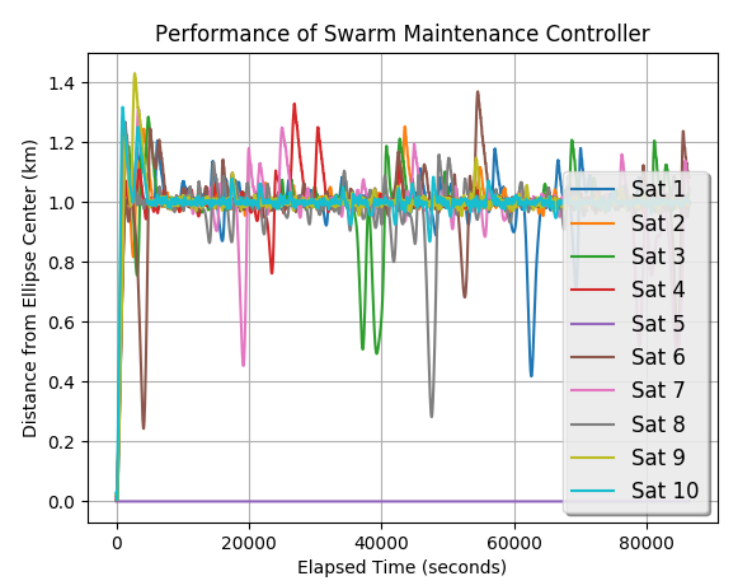

Figure 13. Results from a successful configuration, with $A=B=5000$, show that excursions beyond the spheroid volume boundary are brief and quickly contained.

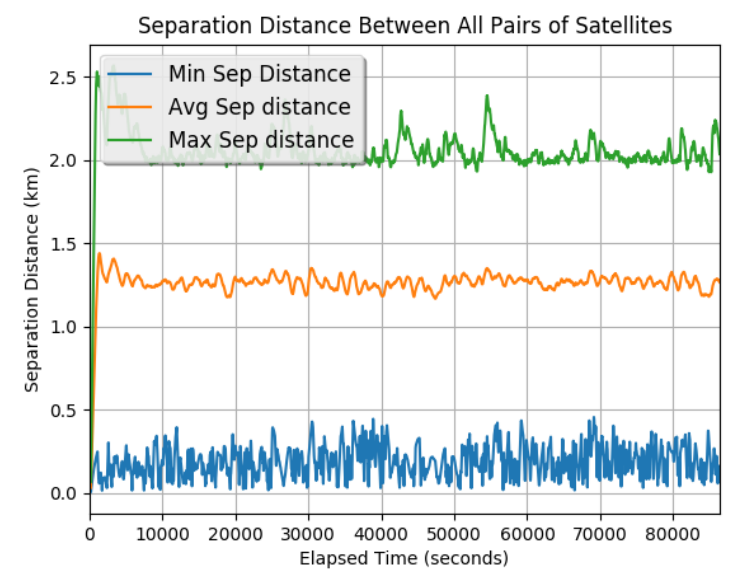

Figure 14. In this summary of the results shown in Figure 13, the maximum separation distance is approximately equal to the diameter of the spheroid boundary.

Propulsion solutions for the APF approach-The APF approach for a volume container swarm type not fuel-optimal and limits the duration of swarm controllability. The current approach as-is can potentially be a method of maintaining a tight swarm of small satellites for a short time (on the order of one day). Because the APF volume container control approach may command the satellite to perform maneuvers very frequently, the attitude and thruster control systems must be fully autonomous. In conventional control approaches, the onboard orbit knowledge will limit how much lead time the spacecraft will have to respond to a predicted event such as a boundary approach. Incorporating time-to-intercept into the guidance algorithm may be a method of fuel-optimization; knowing in advance that a maneuver will be needed would allow for a guidance algorithm to plan a more optimized maneuver. Although a long lead time could conceivably be helpful, it is not strictly necessary. SODA's APF algorithm does not rely on path-planning and long lead times, and instead responds to changes in the artificial potential field.

The results with the current APF implementation show that, of the studied cases, the control authority required is on the order of at least $1 \mathrm{~m} / \mathrm{s}$. Chemical propulsion systems that provide thrust in the $1-5 \mathrm{~N}$ range are a feasible design choice for this swarm type. Bipropellant and non-toxic monopropellant systems have higher specific impulses than cold gas systems and allow the spacecraft to achieve more total impulse, extending the swarm lifetime. The drawback of these systems is their much lower specific impulses compared to electric systems. However, with the studied APF swarm maintenance approach, and under the impulsive burn assumption, no available electric propulsion system can provide enough impulsive thrust to maintain a volume container swarm configuration in any of the deployment configurations. Continuous finite maneuvers with low thrust propulsion systems could be the next step to investigate.

\section{Conclusions And Future Work}

Algorithms that drive satellites to wander within a volumetric boundary may enable novel science and technology applications that are not feasible with more traditional swarm types like in-track or cross-track formations. This paper studied two approaches to containing small satellites within a delimited volume container. We considered a LEO swarm mission concept in which $106 \mathrm{U}$ CubeSats were contained within a spheroid volume boundary.

The first approach counteracted the deployment vector in order to prevent the total dispersion of the swarm. Prescribed maneuvers allowed the satellites to stay in quasi-periodic ranges with respect to the reference point of the swarm, after applying a maneuver to minimize the free drift. The representative rotational deployment case showed that the Y-Z plane components affect the swarm controlability in terms of amplitude of oscillation in some cases. Also, the deployment in the velocity direction requires an order of magnitude more than the rest due to the higher dispersion in $\Delta \mathrm{V}$ magnitude around that axis. State of the art propulsion systems for CubeSats meet the requirements required for swarm maintenance with this algorithm.

Next, we sought a more random distribution of the spacecraft, and studied mission design using APFs as controllers. Numerical experimentation included varying the repulsive potential functions, spheroid radius, maximum $\Delta \mathrm{V}$ allowed per maneuver, and the duty cycle at which the maneuvers can take place. Only a small portion of the studied trade space is successful. The test cases that met our defined success criteria were generally characterized by a spheroid radius of $1 \mathrm{~km}$, a value of 500-1000 of the tuning parameters, a duty cycle of 10 or $30 \mathrm{~s}$, and a burn size of $1 \mathrm{~m} / \mathrm{s}$ per impulsive maneuver. Suitable COTS propulsion systems that can provide these capabilities for a $6 \mathrm{U}$ CubeSat are limited to chemical systems that can provide high thrust impulsive maneuvers.

In some scenarios, it can require more time for the swarm to be set with the deployment control in comparison with the APF that takes a more radical and quicker implementation. Also, a swarm that is set with the APF approach implies a more distributed and chaotic behavior of its members that may be certainly more interesting for particular applications that could require the satellites to wander chaotically around a point in space in a responsive manner. The first approach is much more predictable, in which the satellites follow a quasi- 
periodic motion respect to the reference point of the swarm. The advantage in this case is that the required $\Delta \mathrm{V}$ to achieve a sustainable swarm configuration is much more affordable. In the case without differential drag involved, the $\Delta \mathrm{V}$ required is as small as the difference in the differential velocity component of the deployment vectors of each satellite with respect to the reference point.

The simulated test cases presented here will serve as a basis for future swarm mission design efforts. Continued trade space study and algorithm development is necessary to reduce the total $\Delta \mathrm{V}$ required for implementation of the APF approach, and therefore to enable CubeSat science missions. Specifically, applying optimal control methods to find fueloptimal maneuvers will be studied next. Other active research efforts include propellantless swarm control via differential drag, and autonomous control via low-thrust, finite maneuvers. As interest in swarm mission applications grows, so too will CubeSat capabilities, enabling novel science objectives in the future.

\section{ACKNOWLEDGMENTS}

The authors of this paper would like to thank the support from the management of the Mission Design Division at the NASA Ames Research Center.

\section{REFERENCES}

[1] Conn T., Plice L., Dono A., Ho M., "Operating Small Sat Swarms as a Single Entity: Introducing SODA", USU Small Satellite Conference, Logan, UT, 2017

[2] Ismael Lopez and Colin R. McInnes. "Autonomous rendezvous using artificial potential function guidance", Journal of Guidance, Control, and Dynamics, Vol. 18, No. 2 (1995), pp. 237-241.

[3] D. P. Scharf, F. R. Hadaegh, and S. R. Ploen, "A Survey of Spacecraft Formation Flying Guidance and Control (Part I): Control," Proceedings of the American Control Conference, Vol. 2, Inst. of Electrical and Electronics Engineers, Piscataway, NJ, 2004, pp. 2976-2985.

[4] Clohessy, W.H. and Wiltshire, R.S., "Terminal Guidance System for Satellite Rendezvous,'Journal of the Aerospace Sciences, Vol. 27, No.0, Sep, 1960, pp.653658,674 .

[5] R. H. Vassar R. B. Sherwood "Formationkeeping for a Pair of Satellites in a Circular Orbit" AIAA Journal of Guidance Control and Dynamics, vol. 8 pp. 235-242 1985.

[6] D. P. Scharf, F. R. Hadaegh, and S. R. Ploen, "A Survey of Spacecraft Formation Flying Guidance and Control (Part I): Guidance," Proceedings of the American Control Conference, Inst. of Electrical and Electronics Engineers, Piscataway, NJ, 2003, pp. 17331739.

[7] Hughes, S.,Formation Tetrahedron Design for Phase 1 of the Magnetospheric Multiscale Mission, Flight Dynamics Analysis Branch, NASA Goddard Space Flight Center, 2004.

[8] V Kapila, AG Sparks, JM Buffington, Q Yan, "Spacecraft Formation Flying: Dynamics and Control", AIAA Journal of Guidance, Control, and Dynamics vol. 23, pp. 561-564.

[9] D.C. Redding, N.J. Adams, and E.T. Kubiak, "Linear-
Quadratic Stationkeeping for the SYS Orbiter,'Journal of Guidance, Control, and Dynamics, vol. 12, 1989, pp.248255.

[10] James K., Bodnar M., Freedman M., Osborne L., Grist R., Hoyt R., "Hydros: High performance waterelectrolysis propulsion for cubesats and microsats", AAS 17-145.

[11] VACCO Industries, "CubeSat Propulsion Systems.” Internet: www.cubesat-propulsion.com, 2012 [Oct. 10, 2017].

[12] GOMspace, MEMS Cold Gas Propulsion Module for $6 \mathrm{U}_{+}$nanosatellites. Internet: gomspace.com/Shop/subsystems/propulsion/nanoprop6u-propulsion.aspx, 2017 [Oct. 19, 2017].

[13] Enpulsion, The IFM Nano Thruster. Internet: enpulsion.com, 2017 [Oct. 19, 2017].

[14] Accion Systems, A New Ion Engine. Internet: accionsystems.com, 2017 [Oct. 19, 2017].

[15] Dinardi A., Anflo K., Friedhoff P., ”On-Orbit Commissioning of High Performance Green Propulsion (HPGP) in the SkySat Constellation", SmallSat Conference, Logan, Utah, 2017.

[16] Taylor, C.J., and Kiregman D. J., Minimization on the Lie Group SO(3) and Related Manifolds, Technical Report No. 9405. Yale University, April, 1994.

\section{BIOGRAPHY}

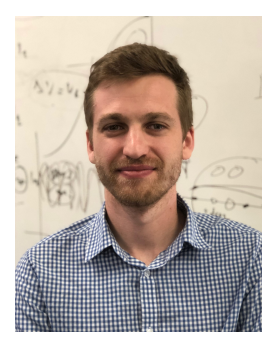

Andres Dono received his B.S. and M.S. degrees in Physics from the University of Santiago de Compostela, and a MSc in Space Studies from ISU. He is currently a flight dynamics and systems engineer working for Millennium Engineering and Integration Services, at NASA Ames Research Center.

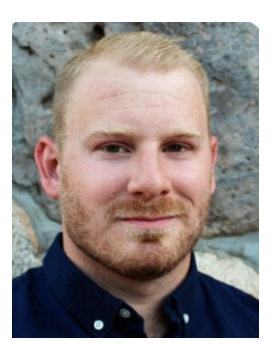

Joel Mueting received his B.S. degree in Aerospace Engineering in 2014 and an M.S. in Systems Engineering from the University of Arizona in 2016. He is currently a Flight Dynamics engineer at NASA Ames Research Center.

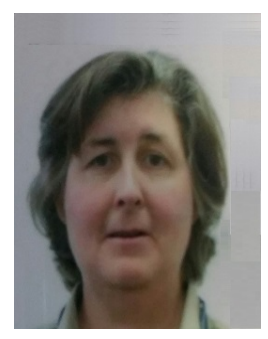

Laura Plice received her degrees in Aerospace Engineering from the University of Michigan, completing her MS in 1985. She was the Flight Dynamics lead on the Lunar Atmosphere and Dust Environment Explorer and is currently leading the Flight Dynamics team in the Mission Design Division at NASAs Ames Research Center and on the Arcus MIDEX Step 1 project. 


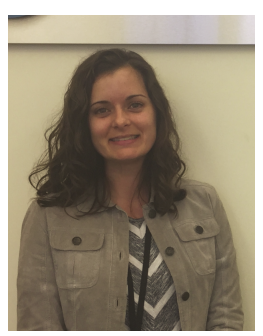

Tracie Conn is an engineer in the Mission Design Division at NASA Ames Research Center, with a research focus on satellite swarm missions. She received a bachelors degree from the University of Texas at Austin and a PhD from the University of Texas at Arlington, both in aerospace engineering.

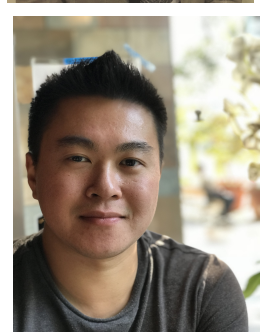

Michael Ho received his B.S. degree in Computer Science in 2016 from San Jose State University. He is currently a computer scientist at Stinger Ghaffarian Technologies, under a contract with NASA Ames Research Center. 